\title{
General Psychiatry Rates and risk factors for suicidal ideation, suicide attempts and suicide deaths in persons with HIV: a systematic review and meta-analysis
}

\author{
Matt Pelton, ${ }^{1}$ Matt Ciarletta (D) ${ }^{1}$ Holly Wisnousky, ${ }^{1}$ Nicholas Lazzara, ${ }^{1}$ \\ Monica Manglani, ${ }^{1}$ Djibril M Ba, ${ }^{1}$ Vernon M Chinchillii, ${ }^{1}$ Ping Du, ${ }^{1}$ \\ Anna E Ssentongo, ${ }^{1,2}$ Paddy Ssentongo (D) 1,3
}

To cite: Pelton M, Ciarletta M, Wisnousky $\mathrm{H}$, et al. Rates and risk factors for suicidal ideation, suicide attempts and suicide deaths in persons with HIV: a systematic review and metaanalysis. General Psychiatry 2021;34:e100247. doi:10.1136/ gpsych-2020-100247

- Additional material is published online only. To view, please visit the journal online (http://dx.doi.org/10.1136/ gpsych-2020-100247).

MP and MC contributed equally.

Received 24 April 2020

Revised 18 December 2020

Accepted 04 February 2021

\section{Check for updates}

(C) Author(s) (or their employer(s)) 2021. Re-use permitted under CC BY-NC. No commercial re-use. See rights and permissions. Published by BMJ.

For numbered affiliations see end of article.

Correspondence to Dr Paddy Ssentongo; pssentongo@pennstatehealth. psu.edu

\section{ABSTRACT}

Background People living with HIV/AIDS (PLWHA) must contend with a significant burden of disease. However, current studies of this demographic have yielded wide variations in the incidence of suicidality (defined as suicidal ideation, suicide attempt and suicide deaths). Aims This systematic review and meta-analysis aimed to assess the lifetime incidence and prevalence of suicidality in PLWHA.

Methods Publications were identified from PubMed (MEDLINE), SCOPUS, OVID (MEDLINE), Joanna Briggs Institute EBP and Cochrane Library databases (from inception to before 1 February 2020). The search strategy included a combination of Medical Subject Headings associated with suicide and HIV. Researchers independently screened records, extracted outcome measures and assessed study quality. Data were pooled using a random-effects model. Subgroup and metaregression analyses were conducted to explore the associated risk factors and to identify the sources of heterogeneity. Main outcomes were lifetime incidence of suicide completion and lifetime incidence and prevalence of suicidal ideation and suicide attempt.

Results A total of 185199 PLWHA were identified from 40 studies (12 cohorts, 27 cross-sectional and 1 nested case-control). The overall incidence of suicide completion in PLWHA was $10.2 / 1000$ persons (95\% Cl: 4.5 to 23.1 ), translating to 100 -fold higher suicide deaths than the global general population rate of $0.11 / 1000$ persons. The lifetime prevalence of suicide attempts was 158.3/1000 persons (95\% Cl: 106.9 to 228.2) and of suicidal ideation was $228.3 / 1000$ persons $(95 \% \mathrm{Cl}: 150.8$ to 330.1$)$. Metaregression revealed that for every 10-percentage point increase in the proportion of people living with HIV with advanced disease (AIDS), the risk of suicide completion increased by 34 per 1000 persons. The quality of evidence by Grading of Recommendations, Assessment, Development and Evaluations for the suicide deaths was graded as 'moderate' quality.

Conclusions The risk of suicide death is 100 -fold higher in people living with HIV than in the general population. Lifetime incidence of suicidal ideation and attempts are substantially high. Suicide risk assessments should be a priority in PLWHA, especially for those with more advanced disease.

\section{INTRODUCTION}

Since its discovery in the 1980s, HIV continues to carry a significant global burden of disease. While the disease remains incurable, highly active antiretroviral therapy (HAART) has been effective in controlling disease progression, improving quality of life and prolonging longevity. ${ }^{1}$ In 2017, the Global Burden of Disease approximated that globally, 36.8 million people were living with HIV/AIDS (PLWHA) ${ }^{23}$ HIV caused approximately one million deaths worldwide and was responsible for the annual 48 disability-adjusted life years (DALYs) per 100000 population. $^{24}$ While The Joint United Nations Programme on HIV and AIDS (UNAIDS) and the WHO provide an effective framework in controlling HIV infection, the current strategies fail to adequately address interventions for the psychosocial burden experienced by PLWHA.

Since the introduction of HAART in 1996, morbidity and mortality have declined in PLWHA, ${ }^{5}$ although the relationship between HAART and suicide risk remains unclear. A longitudinal study followed 163 PLWHA for 2 years and found that HAART increased CD4 counts indicative of immunological rebound and decreased depressive symptoms with a temporal relationship. ${ }^{6}$ However, other studies have suggested that HAART with efavirenz can induce a neuropsychiatric reaction, potentially increasing depressive symptoms and suicide risk. ${ }^{7-9}$ Despite the improved prognosis of HIV, studies continue to find a wide variation in incidence of increased suicidality among PLWHA. ${ }^{1011}$ Marzuk and colleagues found that nearly $9 \%$ of suicide victims had HIV in New York City. ${ }^{12}$ Likewise, a cross-sectional study found that $78 \%$ of women with HIV had suicidal thoughts, and $26 \%$ had attempted suicide since their HIV diagnosis. ${ }^{13}$ Data thus far have shown 
that patient suicide rates within the first year of HIV diagnosis exceed that of the general population. ${ }^{14-16}$ While the factors leading to suicide may mirror those seen in depression, identifying the risks correlated to suicidality in patients with HIV will inform effective preventative measures against suicide. Furthermore, as discussed above, identifying risk factors of suicidal behaviour can improve HIV management in at-risk populations. Currently, it is not well established whether: (1) HIV infection itself increases suicide risk; (2) if HAART increases suicide risk because of side effects; (3) other cofactors that are commonly seen in the HIV population such as depression, lack of social support, stigma, loneliness and so on could affect the suicide risk.

To date, there is no systematic review and meta-analysis of the pooled lifetime incidences of suicide in PLWHA and examined associated risk factors. This has, in part, been confounded by the methodological limitations of different studies leading to a wide variety of incidence reports. ${ }^{17}$ To fill the knowledge gap, we aimed to explore the relationship between HIV/AIDS and suicide risk. We accomplished this goal by conducting a meta-analysis of published literature. The primary objective was to examine the lifetime incidence of suicide completion in PLWHA and delineate the associated risk factors. Furthermore, we examine the lifetime incidence and prevalence of suicidal ideation and attempts within PLWHA. This comprehensive statistical review of the published literature provides a deeper understanding of the effects of HIV on suicide risk.

\section{METHODS}

\section{Database searches, search strategy and terms}

This study has been registered with PROSPERO (registration number: CRD42020161501) and the protocol is published. ${ }^{18}$ This study is being reported per the reporting guidance provided in the Preferred Reporting Items for Systematic Reviews and Meta-Analyses (PRISMA) statement (see online supplemental table 1). ${ }^{17}$ We used the study protocol (online supplemental text 1). We searched PubMed (MEDLINE), SCOPUS, OVID (MEDLINE), Joanna Briggs Institute EBP and Cochrane Library databases to identify studies reporting suicide rates in PLWHA that have been published from inception to before 1 February 2020. Our keyword search was based on Medical Subject Headings (MeSH) with various combinations of 'Suicide*', OR'Depression*', OR'Suicide Attempt*', OR'Suicidal Ideation*', OR'Suicide Completion*' OR'Mental Illness*' OR 'Anxiety*', AND 'HIV*' OR'Human Immunodeficiency Virus', 'AIDS' OR ‘Acquired Immunodeficiency Syndrome'. The full list of search terms is given in online supplemental table 2.

\section{Eligibility criteria}

Studies were selected according to the following criteria: participants, condition or outcome(s) of interest, study design and context.

1. Participants (population): We included studies involving children, adolescents and adult patients living with
HIV (regardless of age or sex). Studies not conducted in humans were excluded.

2. Condition or outcome(s) of interest: The primary outcome is the incidence of suicidality outcome indicating the rate of new (or newly diagnosed) cases of suicidal ideations, suicide attempts or suicide deaths in people living with HIV. It is generally reported as the number of new cases occurring within a period of time (eg, per month or per year) or as a fraction of the population at risk of developing the outcome (eg, new cases per 1000 or 10000 ). We used author-reported definitions according to accepted diagnostic criteria. Secondary outcomes are the risk factors associated with suicidality outcome (eg, HIV viral lead, CD4 T cell count, age, gender and race, major depression, alcohol or drug abuse and dependence, panic disorder, social phobia and schizophrenia).

3. Study design and context: Eligible studies were randomised trials, observational cohort (prospective or retrospective) and cross-sectional studies reporting outcome data and conducted in a wide range of PLWHA. We excluded case series and case reports. No limitations were imposed during the study conduct period and language of publication. Reviews, commentaries and conference/meeting abstracts were excluded.

\section{Data extraction}

Data were extracted from studies using an adapted version of a standard data entry electronic form (table 1). Full-text articles were downloaded and independently reviewed by HW, MP, NL and MC to determine eligibility for inclusion in the analysis. If eligible, data were extracted. Disagreements between extractors were discussed with a fifth author (PS), and a consensus was reached. The incidences of suicidal ideation, attempts and completion were extracted from each publication using a structured data collection spreadsheet. Study-level characteristics included were the year of publication, year or years of study, study methods, mean/median age, gender proportion, proportion on HAART, the proportion with depression and average CD4 count. Studies that do not include enough information to calculate primary outcome (incidence of suicidal ideation, suicide attempt and suicide deaths) were excluded.

\section{Assessment of methodological quality of the papers}

Two authors (MP and MC) independently assessed the quality of the papers included in the review. Assessment of methodological quality was conducted using the Newcastle-Ottawa Quality Assessment Scale, a validated tool for assessing cross-sectional, case-control and cohort studies. ${ }^{19}$ Scores of 8 to the maximum score of 9 were defined as high quality; scores of 5-7 were defined as intermediate quality and scores of 1-4 were defined as low quality. Discrepancies in scoring were resolved by discussion with a third author (PS). Studies were included regardless of the risk of bias and quality scores, but 
Table 1 Study-level characteristics of the articles included in the meta-analysis

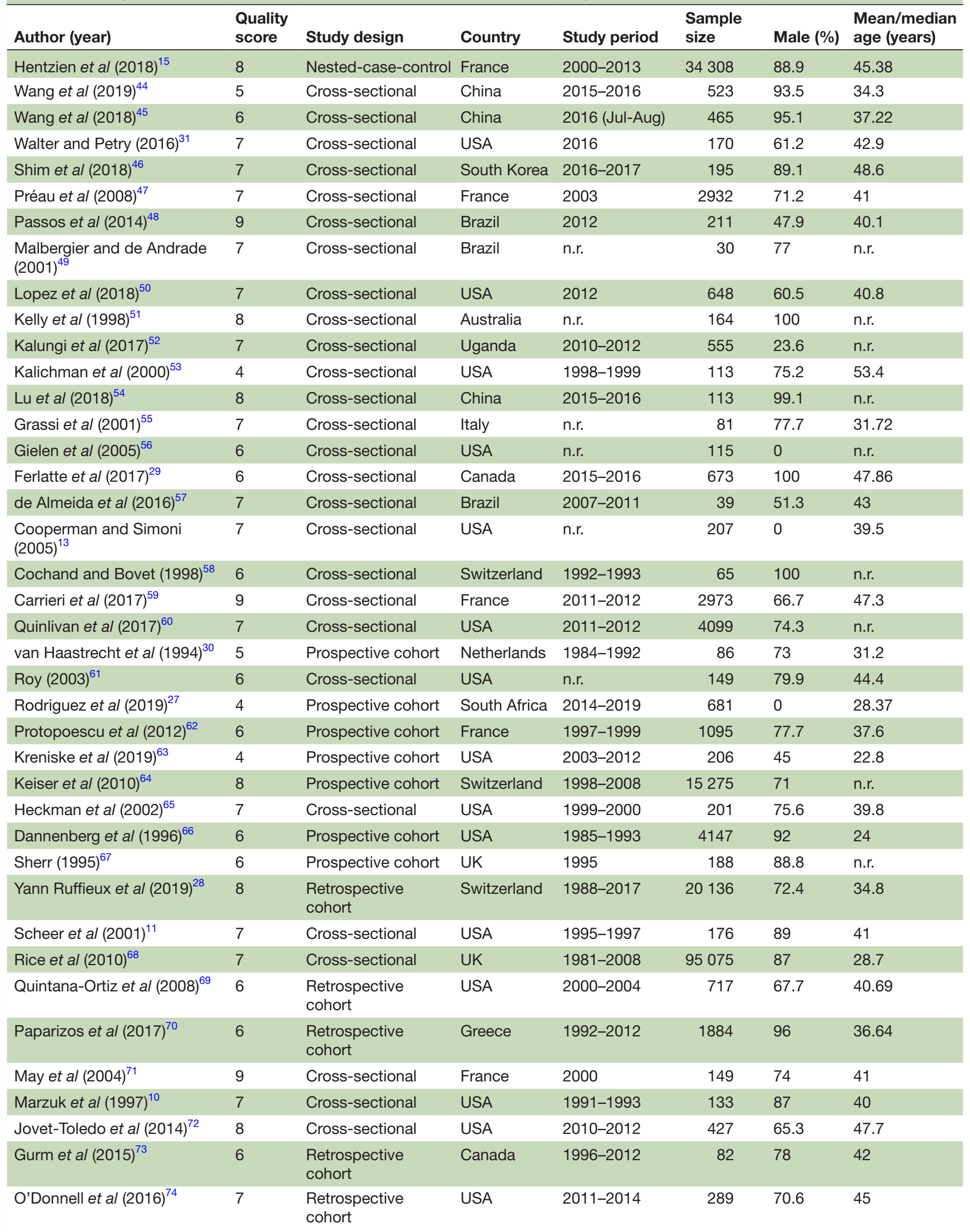

n.r., not reported. 
subgroup analysis was conducted to ascertain the impact of their inclusion (table 1).

\section{Statistical analyses}

We adopted a narrative approach describing the number of studies, study design, country where the studies were conducted and suicidality diagnostic criteria. We examined three outcomes separately. The first was the lifetime incidence of suicide completion (deaths), the second and third were suicide attempts and suicidal ideation in PLWHA. The metaprop and metagen functions from the $\mathrm{R}$ package meta were used to calculate the pooled effect estimates using random-effects models. ${ }^{20}$ Random-effect models were built using a generalised linear mixedeffects model with logit transformation of proportions for pooling of studies. ${ }^{21}$ The CIs were calculated using the exact binomial (Clopper-Pearson) interval method. We applied the DerSimonian and Laird random-effects method to estimate the pooled between-study variance (heterogeneity). ${ }^{22}$ Individual and pooled estimates were graphically displayed using forest plots. A random-effects model assumes the observed estimates can vary across studies because of real differences in the effect in each study as well as sampling variability (chance). Betweenstudy heterogeneity was assessed using $I^{2}$ statistics, expressed as a percentage (low (25\%), moderate $(50 \%)$ and high (75\%)) and a Cochrane's $Q$ statistic (significance level $<0.05) .{ }^{23}$ We assessed the quality of evidence (QoE) using the GRADE (Grading of Recommendations, Assessment, Development and Evaluations) framework using four levels of quality of evidence: very low, low, moderate and high. ${ }^{24}$ The following domains were used for the assessment: risk of bias, imprecision, inconsistency, indirectness and publication bias. ${ }^{25}$ We reported the overall strength of evidence of the outcome of interest. Potential sources of heterogeneity were investigated further by subgroup or meta-regression analyses according to baseline characteristics and methodological covariates. Additionally, we explored geographical differences in suicide risk. Meta-regression analysis was conducted using the following covariates as regressors: study-level median or mean age (also dichotomised as mean/median age $\geq 40$ years or $<40$ years), study-level gender proportions, year of study, depression proportions, the proportion of study population with AIDS and patients on HAART, mean/ median CD4 count, percentage of the study population with a depression diagnosis and study quality score. Potential ascertainment bias (as might be caused by publication bias) was assessed with funnel plots, by plotting the study effect size against SEs of the effect size and Egger's test. ${ }^{26}$ We report absolute differences (per 1000) in the overall rates of suicide.

\section{RESULTS}

Our search retrieved 1518 articles, of which 539 full-texts were carefully reviewed and considered as potentially relevant (PRISMA; figure 1). The full-text review identified
110 records identified from PubMed (MEDLINE), 1402 records identified from SCOPUS, 6 records identified from OVID (MEDLINE)/OVID (JSTOR), 0 records identified from Joanna Briggs Institute, and 0 records from Cochrane Library.

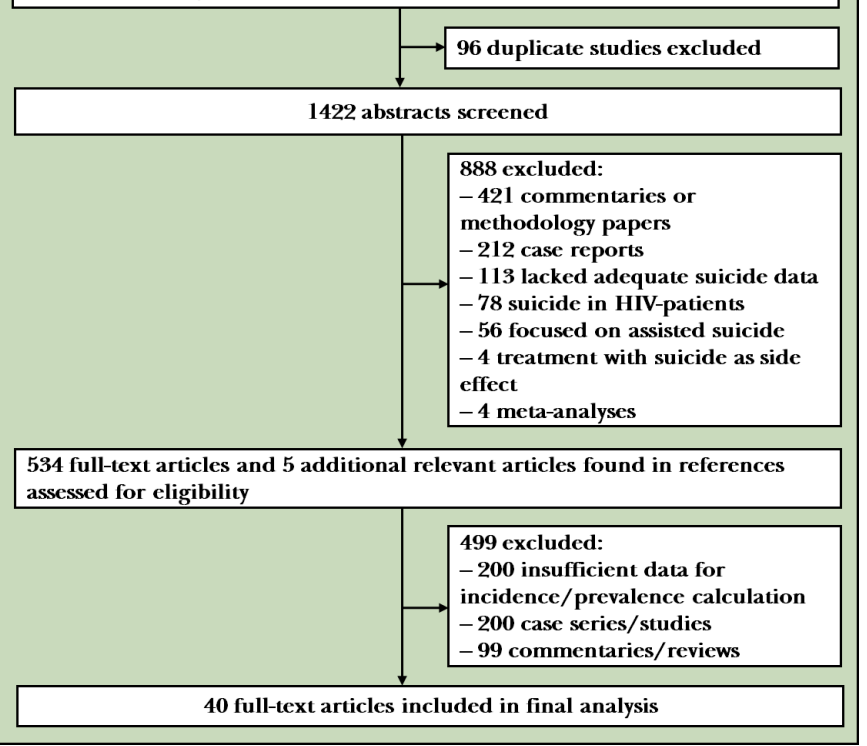

Figure 1 PRISMA flowchart for the meta-analysis of suicidality in PLWHA. Forty full-text articles were incorporated into the review from a total of 1518 titles. PLWHA, people living with HIV/AIDS.

40 eligible articles from 14 countries (table 1): USA 15, France 5, Brazil 3, Switzerland 3, South Africa 1, China 3, Canada 2, Australia 1, Italy 1, South Korea 1, Greece 1, Uganda 1, UK 2 and Netherlands 1. Twenty-seven studies were cross-sectional, while five were retrospective cohorts, seven were prospective cohorts and one was a nested case-control study. Source of participants varied widely between studies from specific subgroups such as pregnant and postpartum women to national databases. ${ }^{11} 2728$ Diagnostic criteria for suicidal ideation and attempts were clinical and assessed by various questionnaires, both formal and informal (eg, MINI, Beck Depression Inventory-II or asking if participants had 'considered ending their lives'). ${ }^{29}$ Illness duration varied widely between studies, from immediate postdiagnosis period to lifetime risk. ${ }^{30}$ Suicide completion was reported as the intentional self-infliction of death. There was high heterogeneity in the diagnostic methods used to identify suicide attempts and ideation. The articles included in the final selection comprised a total of 185199 PLWHA. The articles that reported suicide completion comprised a total of 177748 PLWHA, and the cumulative number of patients with suicide completion was 973 . The median age of the patients included in the study was 39 years. The quality of evidence by Grading of Recommendations, Assessment, Development and Evaluations for the suicide deaths was graded as 'moderate' quality (online supplemental table 3 ). The median study quality score was 7 out of 9 (range from 4 to 9 , table 1 and online supplemental table 3). Study-specific details and references are given in table 1 . 


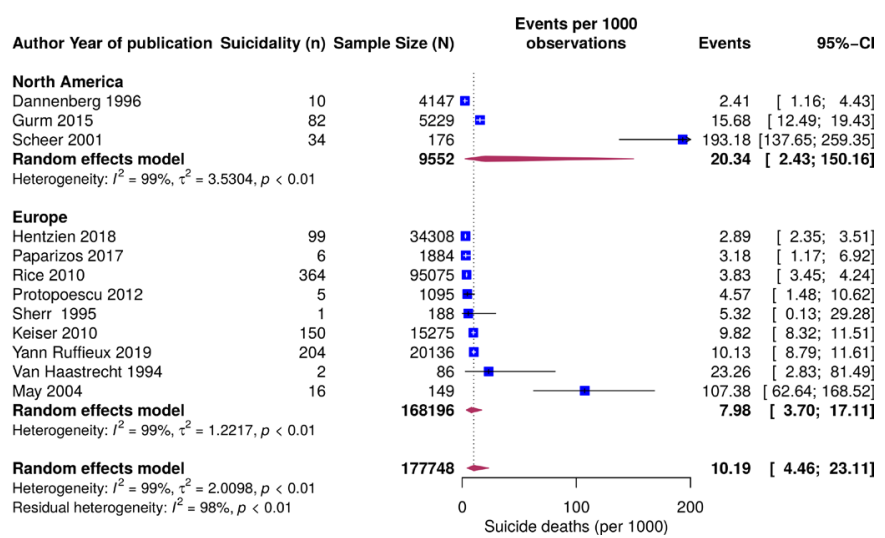

Figure 2 Forest plot of the incidence of suicide deaths per 1000 PLWHA by continent from random-effects model: event values represent the number of suicide deaths per 1000 PLWHA $(95 \% \mathrm{Cl})$. Blue squares and their corresponding lines are the point estimates and $95 \% \mathrm{Cls}$ per study. Maroon diamonds represent the pooled estimate of the prevalence for each subgroup (width denotes $95 \%$ Cls). Heterogeneity by continent: North America $\left(\mathrm{I}^{2}=99 \%\right)$; Europe $\left(\mathrm{I}^{2}=99 \%\right) ; \mathrm{p}$ value for the interaction comparing the different subgroups is 0.41 . PLWHA, people living with HIV/AIDS.

\section{Pooled incidence of suicide deaths in PLWHA}

The pooled incidence of suicide completion was 10.2 per 1000 population (95\% CI: 4.5 to 23.1) (figure 2). Betweenstudy heterogeneity for the cumulative incidence was large $\left(\mathrm{I}^{2}=99 \% ; \mathrm{p}<0.01\right)$. Only North American and European studies reported suicide completion. We carried out subgroup analysis by continent to explore regional differences in suicide completion. The pooled incidence of suicide in studies conducted in North America was twice as high as that in Europe, although the difference was not statistically significant. Egger's test for publication bias was significant $(\mathrm{p}<0.001)$ and funnel plots displayed asymmetry (online supplemental figure 1).

\section{Pooled incidence and prevalence of suicide attempts in PLWHA}

The overall pooled incidence of suicide attempts per 1000 population was 20.4 (95\% CI: 2.4 to 154.9) (figure 3). The incidence in North America was 50 times as high compared with that in Europe. Likewise, in PLWHA, the lifetime prevalence of suicide attempts was 158.3 per 1000 persons (95\%CI: 106.9 to 228.2). The prevalence was higher in the Americas and Australia than in Africa and Asia (figure 4). Displayed in online supplemental figure 2 are the country-specific prevalence of suicide attempts. Egger's test for publication bias was significant $(p<0.001)$ and funnel plots displayed asymmetry (online supplemental figure 3 ).

\section{Pooled prevalence of suicidal ideation in PLWHA}

The overall pooled lifetime prevalence of suicidal ideation per 1000 population was 228.3 (95\%CI: 150.8 to 330.1) (figure 5). Africa and Europe displayed lower prevalence in suicidal ideation compared with that in North

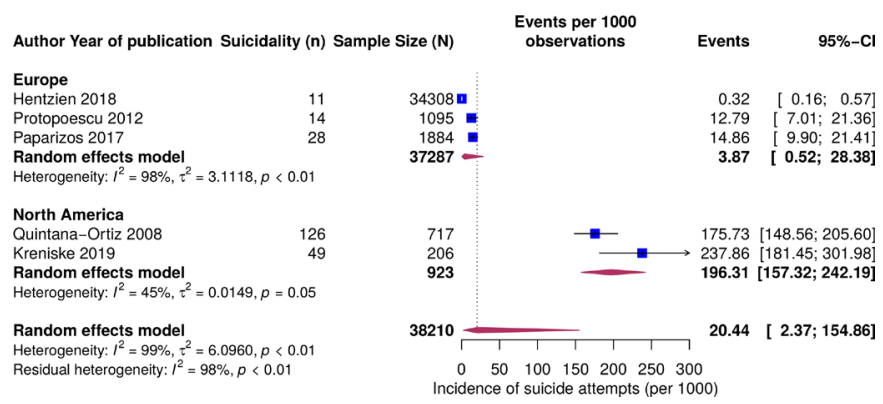

Figure 3 Forest plot of the incidence of suicide attempts per 1000 PLWHA by continent from random-effects model: event values represent the number of new cases of suicide attempts per 1000 PLWHA $(95 \% \mathrm{Cl})$. Blue squares and their corresponding lines are the point estimates and $95 \% \mathrm{Cls}$ per study. Maroon diamonds represent the pooled estimate of the prevalence for each subgroup (width denotes 95\% confidence intervals). Heterogeneity by continent: North America $\left(I^{2}=45 \%\right)$; Europe $\left(l^{2}=98 \%\right)$; p value for the interaction comparing the different subgroups is $<0.0001$. PLWHA, people living with HIV/AIDS.

America, South America and Asia. However, these differences were not statistically significant.

\section{Meta-regression}

To explore sources of heterogeneity in suicidality, we conducted meta-regression analysis with the following covariates: study-level median or mean age (also dichotomised as mean/median age $\geq 40$ years or $<40$ years), gender (proportions), year of study, major depression (proportions), AIDS and HAART (proportions), CD4 count (median), depression (proportions) and study quality (high versus low/medium). Summarised in online supplemental table 4 are meta-regression results. The study-level frequency of AIDS was significantly associated with the risk of suicide completion. Per 10-percentage point increase in people living with AIDS, the risk of completing suicide increased by 34 per 1000 persons. The increasing year of the study and increasing studylevel mean CD4 count were associated with a lower risk of suicide. However, the associations were not statistically significant $(p=0.81$ and $p=0.32$, respectively). High study quality, male gender and increasing age were nonsignificant risk factors of suicide completion.

On the other hand, no sociodemographic or clinical factors were significantly associated with suicide attempts. HAART use was protective but had wider CI. Per 1-percentage point increase in the PLWHA on HAART, 14 per 1000 persons less had attempted suicide (95\% CI: -48.70 to 21.70 ).

\section{DISCUSSION}

\section{Main findings}

About 40 million people of the global population are currently living with HIV/AIDS. ${ }^{3}{ }^{32}$ The era of HAART treatment has brought significant improvements in patient longevity and quality of life; however, PLWHA 


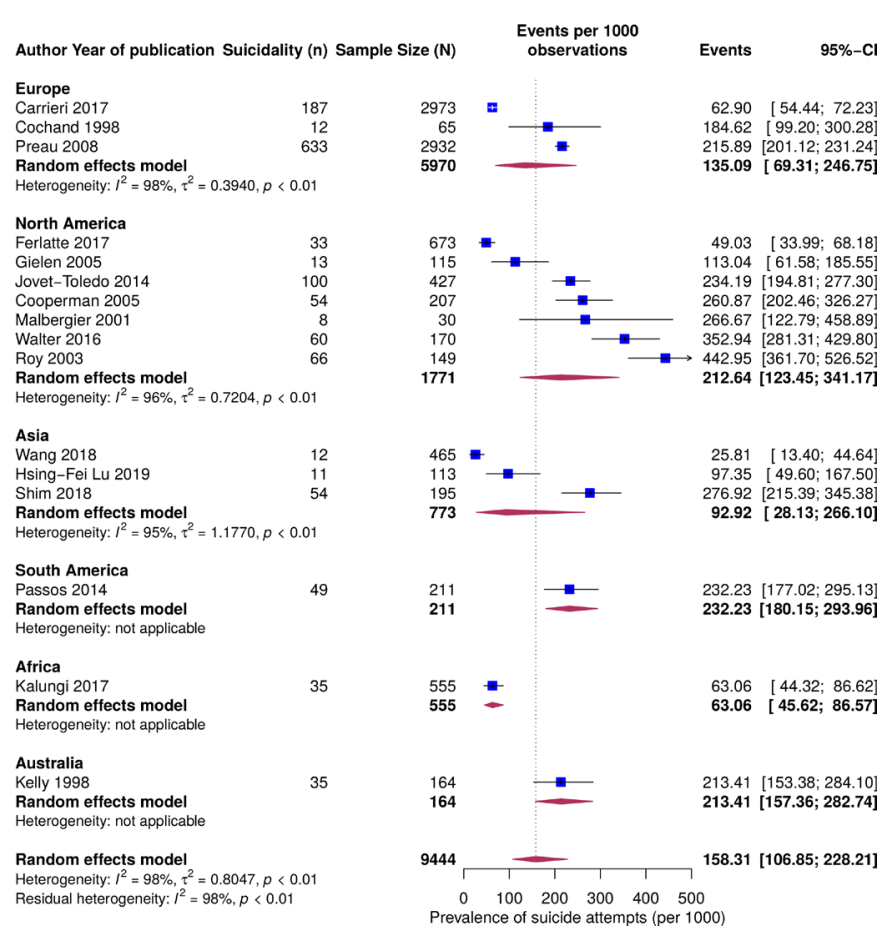

Figure 4 Forest plot of the prevalence of suicide attempts per 1000 PLWHA by continent from random-effects model: event values represent the number of suicide attempts per 1000 PLWHA $(95 \% \mathrm{Cl})$. Blue squares and their corresponding lines are the point estimates and $95 \% \mathrm{Cls}$ per study. Maroon diamonds represent the pooled estimate of the prevalence for each subgroup (width denotes 95\%Cls). Heterogeneity by continent: North America $\left(I^{2}=96 \%\right)$; Europe $\left(I^{2}=98 \%\right)$; Asia $\left(I^{2}=95 \%\right) ;$ Africa $\left(I^{2}=\right.$ not applicable, one study); South America $\left(I^{2}=\right.$ not applicable, one study); Australia $\left(I^{2}=\right.$ not applicable, one study); $p$ value for the interaction comparing the different subgroups is $<0.0001$. PLWHA, people living with HIV/AIDS.

experience a heavy burden of psychosocial conditions that are frequently undiagnosed and untreated. In our study, the pooled incidence of suicide completion among PLWHA globally was 10.2 per 1000 (95\% CI: 4.5 to 23.1), translating to a 100 -fold greater suicide completion rate compared with the global population rate of $0.11 / 1000 .^{33}$ While the suicide completion rate was twice as high in North America (20.4/1000, 95\%CI: 2.43 to 150.16), compared with that in Europe (8.4/1000, 95\% CI: 3.69 to $19)$, this difference was not significant. Importantly, the most striking difference found was between the prevalence of suicide attempts across the geographic regions. While we found a pooled global prevalence of suicide attempts at 158.3/1000 in PLWHA, the pooled prevalence of suicide attempt in this cohort was highest in North America, South America and Australia at 212.6/1000 (95\%CI: 123.5 to 314.2 ), $232.2 / 1000$ (95\%CI: 180.2 to 294.0) and 213.4/1000 (95\%CI: 157.4 to 282.7), respectively. This is in striking comparison to a global lifetime suicide attempt prevalence of $3 \%$ in the general population. ${ }^{34}$ Additionally, the overall pooled prevalence of suicidal ideation in PLWHA was 228.3/1000 (95\%CI: 150.8 to 330.1$)$. This is markedly increased compared with the global suicidal ideation rate of $9 \%$ in the general

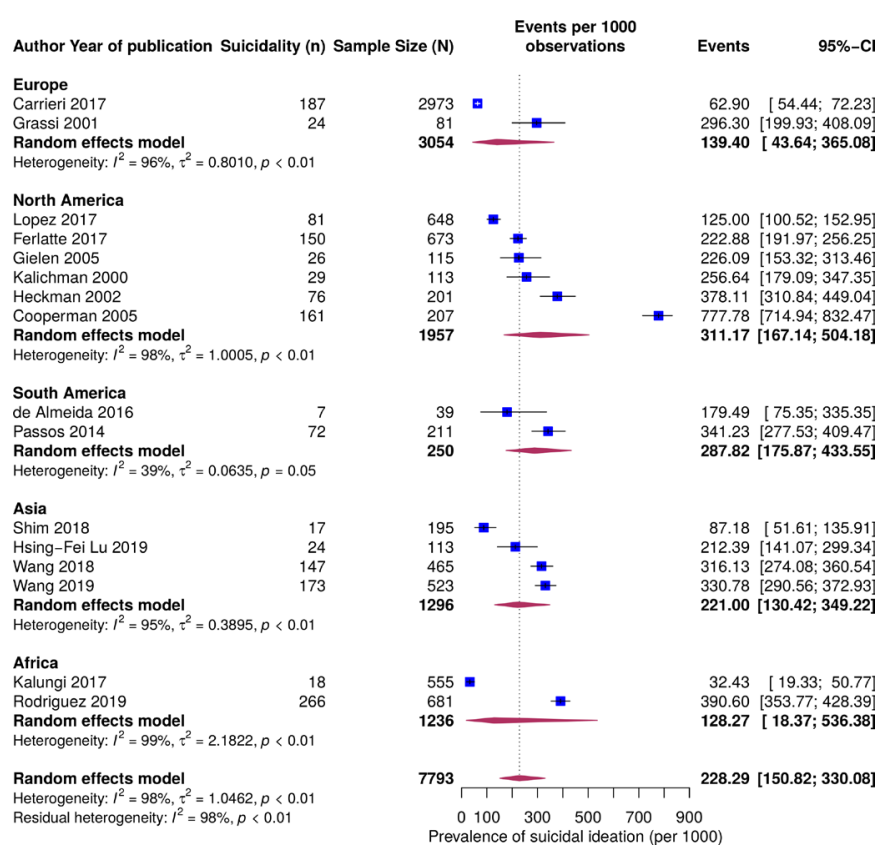

Figure 5 Forest plot of the prevalence of suicidal ideation per 1000 PLWHA by continent from random-effects model: event values represent the number of suicidal ideation per 1000 PLWHA (95\% Cl). Blue squares and their corresponding lines are the point estimates and $95 \% \mathrm{Cls}$ per study. Maroon diamonds represent the pooled estimate of the prevalence for each subgroup (width denotes 95\%Cls). Heterogeneity by continent: North America $\left(I^{2}=98 \%\right)$; Europe $\left(I^{2}=96 \%\right)$; Asia $\left(I^{2}=95 \%\right) ;$ Africa $\left(l^{2}=99 \%\right)$; South America $\left(l^{2}=39 \%\right) ;$ p value for the interaction comparing the different subgroups is 0.56 . PLWHA, people living with HIV/AIDS.

population. ${ }^{34}$ Collectively, these data suggest that PLWHA are at high risk for attempting suicide. Such observation requires appropriate interventions in those at the highest risk.

\section{Strength and limitations}

The major strength of our analysis is the detailed and allinclusive review of literature without limitation to regions that yielded a very large sample of 177748 PLWHA assessed for suicide deaths. The cumulative number of patients with suicide completion of 973 was also large. Despite these strengths, the study had some limitations. First, substantial heterogeneity among the included studies in suicide reporting and overall methodology could have resulted in the variations of the reported suicide outcomes. This heterogeneity includes differences in the sampled period and the reporting of suicide attempts over weeks, months or overall lifetime risk. Second, only English language databases were searched, which would have introduced selection bias in the types of studies included in the analysis. A final notable limitation was the cohort selection within each body of work; some studies limited their work to perinatally infected individuals, pregnant women and/or intravenous drug users. These populations may be at higher suicide risk at baseline. To overcome these limitations, we conducted 
meta-regression to statistically explore the sources of heterogeneity in the outcome of interest.

\section{Implications}

Globally in the general population, one out of every three individuals with suicidal ideation will attempt it, and one out of every 286 attempts will be completed. Our results suggest that in PLWHA for every 2 individuals with suicidal ideations, there is one individual with a suicide attempt, and for every 13 suicide attempts, one person may complete suicide. This is indicative of an increased risk in PLWHA for completed suicide than that of the general population, thus prompting further examination into the characteristics pertinent to these findings.

Determinants for an increased risk of suicide in PLWHA are multifactorial. They include the physiological effects of HAART or decreased CD4 count, neurological symptoms in patients categorised as having neuro HIV, the stigma that is still associated with the disease or the effect of disease on interpersonal relationships. ${ }^{35} 36$ In our meta-regression analysis in which we explored the risk factors of suicide, stage $\mathrm{V}$ disease (AIDS) was significantly associated with the risk of suicide completion. This relationship is not surprising considering that this stage is associated with high a viral load, fostering a direct effect of the virus on the brain. Previous studies have found a higher $\mathrm{CD} 4$ count to have a protective effect on suicide completion. In a recent study exploring seizure frequency in PLWHA, the advanced stage of HIV was significantly associated with new-onset seizures. The author argued that direct brain injury, possibly caused by the virus, could be a potential mechanism of brain injury. ${ }^{37}$ Further work should be conducted to determine the mechanisms by which the progression of HIV modulates the risk of suicide completion.

Of note, data have shown that suicide rates are extraordinarily high in PLWHA within the first year of diagnosis. ${ }^{38}$ Taking this into consideration with our results, there may be a bimodal distribution for excessive suicide risk within the first year of diagnosis and if the disease progresses to stage V. Given our results, we suggest the most effective actionable targets to reduce the rate of suicidal ideation, attempt and completion is immediate and routine suicide risk assessment, psychological counselling and mental health treatment in conjunction with antiviral treatment to maintain or increase CD4 counts. These intervention strategies have been shown to reduce depression and suicide. ${ }^{39}$

Preliminary studies have shown that brief interventions for suicide prevention can be used shortly after diagnosis, which reduces suicidal ideation when compared with standard post-test counselling. ${ }^{40}$ Alternatively, internetbased counselling is effective at reducing depressive symptoms in PLWHA in a randomised control trial in the Netherlands. ${ }^{41}$ Furthermore, several studies have found that spiritual engagement has a protective effect against suicidal ideation in PLWHA within different cultural communities. ${ }^{134243}$ Health providers should thus consider embracing established interventions, encouraging PLWHA to engage with their preferred form of spirituality in a culturally competent manner and treating to increase or maintain CD4 counts to reduce suicidal ideation and completion. Further work should be done to characterise the efficacy of these interventions to reduce suicidal ideation, attempts and completion in PLWHA.

\section{Conclusion}

Within this meta-analysis, we demonstrate for the first time that across an extensive and diverse patient cohort, the rate of suicide deaths in PLWHA is 100-fold higher than the rate that has been reported in the general population. This risk is directly associated with HIV progression; however, antiretroviral treatment and higher CD4 counts seem to be protective against suicide attempts. Lastly, we show that within cohorts of PLWHA, there are regional differences in suicide risk with especially profound rates in North America. We suggest that suicide risk assessment be provided to PLWHA in conjunction with antiviral treatment to improve clinical outcomes, patient longevity and quality of life.

\section{Author affiliations}

${ }^{1}$ Department of Public Health Sciences, The Pennsylvania State University College of Medicine, Hershey, Pennsylvania, USA

${ }^{2}$ Department of Surgery, The Pennsylvania State University College of Medicine, Hershey, Pennsylvania, USA

${ }^{3}$ Center for Neural Engineering, Department of Engineering Science and Mechanics, The Pennsylvania State University, University Park, Pennsylvania, USA

\section{Twitter Paddy Ssentongo @Jeddy007}

Contributors PS: supervised the study. HW, MP, NL and MCC: data collation, analysis, and coauthorship. AS and DMB: data collation and coauthorship. PS and AS: duplicate assessment of study bias and coauthorship. HW, MP, NL, MCC, PS and AS: library search for literature review. PS, VMC and PD: methodology expert and data analysis. PS and MM: drafted the manuscript. All authors have read and approved the final manuscript.

Funding This work was supported by a U.S. National Institutes of Health (NIH) Director's Transformative Award 1R01Al145057 (PS)

Map disclaimer The depiction of boundaries on this map does not imply the expression of any opinion whatsoever on the part of BMJ (or any member of its group) concerning the legal status of any country, territory, jurisdiction or area or of its authorities. This map is provided without any warranty of any kind, either express or implied.

Competing interests None declared.

Patient consent for publication Not required.

Provenance and peer review Not commissioned; externally peer reviewed.

Data availability statement All data relevant to the study are included in the article or uploaded as supplementary information.

Supplemental material This content has been supplied by the author(s). It has not been vetted by BMJ Publishing Group Limited (BMJ) and may not have been peer-reviewed. Any opinions or recommendations discussed are solely those of the author(s) and are not endorsed by BMJ. BMJ disclaims all liability and responsibility arising from any reliance placed on the content. Where the content includes any translated material, BMJ does not warrant the accuracy and reliability of the translations (including but not limited to local regulations, clinical guidelines, terminology, drug names and drug dosages), and is not responsible for any error and/or omissions arising from translation and adaptation or otherwise.

Open access This is an open access article distributed in accordance with the Creative Commons Attribution Non Commercial (CC BY-NC 4.0) license, which permits others to distribute, remix, adapt, build upon this work non-commercially, 
and license their derivative works on different terms, provided the original work is properly cited, appropriate credit is given, any changes made indicated, and the use is non-commercial. See: http://creativecommons.org/licenses/by-nc/4.0/.

\section{ORCID iDs}

Matt Ciarletta http://orcid.org/0000-0002-1576-2708

Paddy Ssentongo http://orcid.org/0000-0003-1565-5731

\section{REFERENCES}

1 Mermin J, Were W, Ekwaru JP, et al. Mortality in HIV-infected Ugandan adults receiving antiretroviral treatment and survival of their HIV-uninfected children: a prospective cohort study. Lancet 2008;371:752-9.

2 Frank TD, Carter A, Jahagirdar D, et al. Global, regional, and national incidence, prevalence, and mortality of HIV, 1980-2017, and forecasts to 2030 , for 195 countries and territories: a systematic analysis for the global burden of diseases, injuries, and risk factors study 2017. Lancet HIV 2019;6:e831-59.

3 WHO U. Joint United nations programme on HIV/AIDS AIDS, 2018. Available: http://aidsinfo.unaids.org/

4 Cassini A, Colzani E, Pini A, et al. Impact of infectious diseases on population health using incidence-based disability-adjusted life years (DALYs): results from the burden of communicable diseases in Europe study, European Union and European economic area countries, 2009 to 2013. Euro Surveill 2018;23.

5 Murphy EL, Collier AC, Kalish LA, et al. Highly active antiretroviral therapy decreases mortality and morbidity in patients with advanced HIV disease. Ann Intern Med 2001;135:17-26.

6 Judd FK, Cockram AM, Komiti A, et al. Depressive symptoms reduced in individuals with HIV/AIDS treated with highly active antiretroviral therapy: a longitudinal study. Aust N Z J Psychiatry 2000;34:1015-21.

7 Prime K, French P. Neuropsychiatric reaction induced by clarithromycin in a patient on highly active antiretroviral therapy (HAART). Sex Transm Infect 2001;77:297-8.

8 Cespedes MS, Aberg JA. Neuropsychiatric complications of antiretroviral therapy. Drug Saf 2006;29:865-74.

9 Mollan KR, Smurzynski M, Eron JJ, et al. Association between efavirenz as initial therapy for HIV-1 infection and increased risk for suicidal ideation or attempted or completed suicide: an analysis of trial data. Ann Intern Med 2014;161:1-10.

10 Marzuk PM, Tardiff K, Leon AC, et al. Hiv seroprevalence among suicide victims in New York City, 1991-1993. Am J Psychiatry 1997;154:1720-5.

11 Scheer S, McQuitty M, Denning P, et al. Undiagnosed and unreported AIDS deaths: results from the San Francisco medical examiner. $J$ Acquir Immune Defic Syndr 2001;27:467-71.

12 Marzuk PM, Tardiff K, Leon AC, et al. Hiv seroprevalence among suicide victims in New York City, 1991-1993. Am J Psychiatry 1997;154:1720-5.

13 Cooperman NA, Simoni JM. Suicidal ideation and attempted suicide among women living with HIV/AIDS. J Behav Med 2005;28:149-56.

14 Aldaz P, Moreno-Iribas C, Egüés N, et al. Mortality by causes in HIVinfected adults: comparison with the general population. BMC Public Health 2011;11:300.

15 Hentzien M, Cabie A, Pugliese P, et al. Factors associated with deaths from suicide in a French nationwide HIV-infected cohort. HIV Med 201810.1111/hiv.12633. [Epub ahead of print: 01 Jun 2018].

16 Jia C-X, Mehlum L, Qin P. AIDS/HIV infection, comorbid psychiatric illness, and risk for subsequent suicide: a nationwide register linkage study. J Clin Psychiatry 2012;73:1315-21.

17 Komiti A, Judd F, Grech P, et al. Suicidal behaviour in people with HIV/AIDS: a review. Aust N Z J Psychiatry 2001;35:747-57.

18 Wisnousky $\mathrm{H}$, Lazzara N, Ciarletta M, et al. Rates and risk factors for suicidal ideation, suicide attempts and suicide deaths in persons with HIV: a protocol for a systematic review and meta-analysis. BMJ Open 2021;11:e037154.

19 Peterson J, Welch V, Losos M. The Newcastle-Ottawa scale (NOS) for assessing the quality of nonrandomised studies in meta-analyses. Ottawa: Ottawa Hospital Research Institute, 2011.

20 Schwarzer G, Carpenter JR, Rücker G. Meta-Analysis with R: Springer, 2015

21 Schwarzer G, Chemaitelly H, Abu-Raddad LJ, et al. Seriously misleading results using inverse of Freeman-Tukey double arcsine transformation in meta-analysis of single proportions. Res Synth Methods 2019;10:476-483.

22 DerSimonian R, Kacker R. Random-effects model for meta-analysis of clinical trials: an update. Contemp Clin Trials 2007;28:105-14.
23 Higgins JPT, Thompson SG, Deeks JJ, et al. Measuring inconsistency in meta-analyses. BMJ 2003;327:557-60.

24 Guyatt GH, Oxman AD, Vist GE, et al. Grade: an emerging consensus on rating quality of evidence and strength of recommendations. $B M J$ 2008;336:924-6.

25 Siemieniuk R, Guyatt G. What is grade. British medical Journal best practice. Available: https://bestpracticebmjcom/info/us/toolkit/learnebm/what-is-grade/ [Accessed 10 Jul 2019].

26 Egger M, Davey Smith G, Schneider M, et al. Bias in meta-analysis detected by a simple, graphical test. BMJ 1997;315:629-34.

27 Rodriguez VJ, Mandell LN, Babayigit S, et al. Correlates of suicidal ideation during pregnancy and postpartum among women living with HIV in rural South Africa. AIDS Behav 2018;22:3188-97.

28 Ruffieux Y, Lemsalu L, Aebi-Popp K, et al. Mortality from suicide among people living with HIV and the general Swiss population: 1988-2017. J Int AIDS Soc 2019;22:e25339.

29 Ferlatte O, Salway T, Oliffe JL, et al. Stigma and suicide among gay and bisexual men living with HIV. AIDS Care 2017;29:1346-50.

30 van Haastrecht HJ, Mientjes GH, van den Hoek AJ, et al. Death from suicide and overdose among drug injectors after disclosure of first HIV test result. AIDS 1994;8:1721-5.

31 Walter KN, Petry NM. Lifetime suicide attempt history, quality of life, and objective functioning among HIV/AIDS patients with alcohol and illicit substance use disorders. Int J STD AIDS 2016;27:476-85.

32 UNAIDS. Global HIV \& AIDS Statistics: 2019 Fact Sheet, 2019. Available: https://www.unaids.org/en/resources/fact-sheet

33 Organization WH. Global health Observatory (GHO) data: suicide rates (per 100000 population), 2016. Available: https://www.who.int/ gho/mental_health/suicide_rates/en/

34 Nock MK, Borges G, Bromet EJ, et al. Cross-National prevalence and risk factors for suicidal ideation, plans and attempts. $\mathrm{Br} J$ Psychiatry 2008;192:98-105.

35 Nanni MG, Caruso R, Mitchell AJ, et al. Depression in HIV infected patients: a review. Curr Psychiatry Rep 2015;17:530.

36 Tesfaw G, Ayano G, Awoke T, et al. Prevalence and correlates of depression and anxiety among patients with HIV on-follow up at alert Hospital, Addis Ababa, Ethiopia. BMC Psychiatry 2016;16:368.

37 Ssentongo P. Prevalence and incidence of new-onset seizures and epilepsy in patients with human immunodeficiency virus (HIV): systematic review and meta-analysis. Epilepsy Behav 2019;93:49-55.

38 Croxford S, Kitching A, Desai S, et al. Mortality and causes of death in people diagnosed with HIV in the era of highly active antiretroviral therapy compared with the general population: an analysis of a national observational cohort. Lancet Public Health 2017;2:e35-46.

39 Pence BW, O'Donnell JK, Gaynes BN. Falling through the cracks: the gaps between depression prevalence, diagnosis, treatment, and response in HIV care. AIDS 2012;26:656.

40 Govender RD SL, Esterhuizen T. Brief suicide preventive intervention in newly diagnosed HIV-positive persons. J Psychiatry 2014;17:543-7.

41 van Luenen S, Garnefski N, Spinhoven P, et al. Guided Internetbased intervention for people with HIV and depressive symptoms: a randomised controlled trial in the Netherlands. Lancet HIV 2018;5:e488-97.

42 Zarei $\mathrm{N}$, Joulaei $\mathrm{H}$. The impact of perceived stigma, quality of life, and spiritual beliefs on suicidal Ideations among HIV-positive patients. AIDS Res Treat 2018;2018:6120127.

43 Simoni JM, Martone MG, Kerwin JF. Spirituality and psychological adaptation among women with HIV/AIDS: implications for counseling. J Couns Psychol 2002;49:139-47.

44 Wang W, Chen X, Li S, et al. Cusp catastrophe modeling of suicide behaviors among people living with HIV in China. Nonlinear Dynamics Psychol Life Sci 2019;23:491-515.

45 Wang W, Xiao C, Yao X, et al. Psychosocial health and suicidal ideation among people living with HIV/AIDS: a cross-sectional study in Nanjing, China. PLoS One 2018;13:e0192940.

46 Shim E-J, Lee SH, Kim NJ, et al. Suicide risk in persons with HIV/ AIDS in South Korea: a partial test of the interpersonal theory of suicide. Int J Behav Med 2019;26:38-49.

47 Préau M, Bouhnik A-D, Peretti-Watel P, et al. Suicide attempts among people living with HIV in France. AIDS Care 2008;20:917-24.

48 Passos SMK, Souza LDdeM, Spessato BC. High prevalence of suicide risk in people living with HIV: who is at higher risk? AIDS Care 2014;26:1379-82.

49 Malbergier A, de Andrade AG. Depressive disorders and suicide attempts in injecting drug users with and without HIV infection. AIDS Care 2001;13:141-50.

50 López JD, Shacham E, Brown T. Suicidal ideation persists among individuals engaged in HIV care in the era of antiretroviral therapy. AIDS Behav 2018;22:800-5. 
51 Kelly B, Raphael B, Judd F, et al. Suicidal ideation, suicide attempts, and HIV infection. Psychosomatics 1998;39:405-15.

52 Kalungi A, Seedat S, Hemmings SMJ, et al. Association between serotonin transporter gene polymorphisms and increased suicidal risk among HIV positive patients in Uganda. BMC Genet 2017;18:71.

53 Kalichman SC, Heckman T, Kochman A, et al. Depression and thoughts of suicide among middle-aged and older persons living with HIV-AIDS. Psychiatr Serv 2000;51:903-7.

54 Lu H-F, Chang N-T, Sheng W-H, et al. [Exploring Suicide Ideation and Suicide Attempts Among HIV-Positive Patients During the First Six Months to One Year After Their Diagnosis]. Hu Li Za Zhi 2018;65:60-72.

55 Grassi L, Mondardini D, Pavanati M, et al. Suicide probability and psychological morbidity secondary to HIV infection: a control study of HIV-seropositive, hepatitis $\mathrm{C}$ virus (HCV)-seropositive and HIV/HCV-seronegative injecting drug users. J Affect Disord 2001;64:195-202.

56 Gielen AC, McDonnell KA, O'Campo PJ, et al. Suicide risk and mental health indicators: do they differ by abuse and HIV status? Womens Health Issues 2005;15:89-95.

57 de Almeida SM, Barbosa FJ, Kamat R, et al. Suicide risk and prevalence of major depressive disorder (MDD) among individuals infected with HIV-1 subtype $\mathrm{C}$ versus B in southern Brazil. J Neurovirol 2016;22:789-98.

58 Cochand P, Bovet P. Hiv infection and suicide risk: an epidemiological inquiry among male homosexuals in Switzerland. Soc Psychiatry Psychiatr Epidemiol 1998;33:230-4.

59 Carrieri MP, Marcellin F, Fressard L, et al. Suicide risk in a representative sample of people receiving HIV care: time to target most-at-risk populations (ANRS VESPA2 French national survey). PLoS One 2017;12:e0171645.

60 Quinlivan EB, Gaynes BN, Lee JS, et al. Suicidal ideation is associated with limited engagement in HIV care. AIDS Behav 2017;21:1699-708.

61 Roy A. Characteristics of HIV patients who attempt suicide. Acta Psychiatr Scand 2003;107:41-4. doi:10.1034/j.16000447.2003.02033.x

62 Protopopescu C, Raffi F, Brunet-François C, et al. Incidence, medical and socio-behavioural predictors of psychiatric events in an 11-year follow-up of HIV-infected patients on antiretroviral therapy. Antivir Ther 2012;17:1079-83.

63 Kreniske P, Mellins CA, Dolezal C, et al. Sounding the alarm: perinatally HIV-infected youth more likely to attempt suicide than their uninfected cohort peers. J Adolesc Health 2019;65:702-5.

64 Keiser O, Spoerri A, Brinkhof MWG, et al. Suicide in HIV-infected individuals and the general population in Switzerland, 1988-2008. Am J Psychiatry 2010;167:143-50.

65 Heckman TG, Miller J, Kochman A, et al. Thoughts of suicide among HIV-infected rural persons enrolled in a telephone-delivered mental health intervention. Ann Behav Med 2002;24:141-8.

66 Dannenberg AL, McNeil JG, Brundage JF, et al. Suicide and HIV infection. mortality follow-up of $4147 \mathrm{HIV}$-seropositive military service applicants. JAMA 1996;276:1743-6.

67 Sherr L, Suicide SL. Suicide and AIDS: lessons from a case note audit in London. AIDS Care 1995;7 Suppl 2:S109-16.

68 Rice BD, Smith RD, Delpech VC. Hiv infection and suicide in the era of HAART in England, Wales and Northern Ireland. AIDS 2010:24:1795-7.

69 Quintana-Ortiz RA, Gomez MA, Báez Feliciano DV, et al. Suicide attempts among Puerto Rican men and women with HIV/AIDS: a study of prevalence and risk factors. Ethn Dis 2008;18:S2-219-24.

70 Paparizos V, Triantafyllopoulou I, Kourkounti S, et al. Suicidal behaviour in HIV-infected patients in Greece. Infez Med 2017;25:64-70

71 May T, Lewden C, Bonnet F, et al. [Causes and characteristics of death among HIV-1 infected patients with immunovirologic response to antiretroviral treatment]. Presse Med 2004;33:1487-92.

72 Jovet-Toledo GG, Clatts MC, Rodriguez-Diaz CE, et al. Risk factors for suicide attempts in a clinic-based sample of people living with HIV in Puerto Rico. AIDS Care 2014;26:1032-5.

73 Gurm J, Samji H, Nophal A, et al. Suicide mortality among people accessing highly active antiretroviral therapy for HIV/AIDS in British Columbia: a retrospective analysis. CMAJ Open 2015;3:E140-8.

74 O'Donnell JK, Gaynes BN, Cole SR, et al. Ongoing life stressors and suicidal ideation among HIV-infected adults with depression. $J$ Affect Disord 2016;190:322-8.

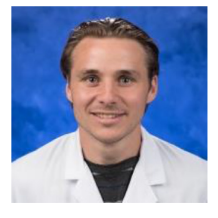

Matt Pelton graduated from Wesleyan University with a bachelor's degree and high honors in the Neuroscience and Behavior major. At present, he is a third year medical student at the Pennsylvania State University College of Medicine in Hershey, Pennsylvania. His main research interests include systematic reviews and metaanalyses across many fields of medicine and anticipates entering the field of Neurology after graduating from medical school.

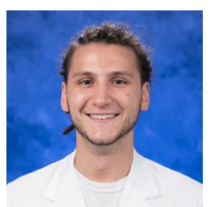

Matthew Ciarletta graduated from York College of Pennsylvania with a major in Biology and a minor in Psychology. At present, he is a third year medical student at the Penn State College of Medicine. His research endeavours include meta-analyses of the psychiatric complications of infectious diseases, as well as behavioural brain mapping in animal models. His research interests centre upon increasing our understanding of the biopsychosocial factors that culminate in the human experience, with a focus on neuropsychological phenomena. 